\title{
P-glycoprotein Function in the Rodent Brain Displays a Daily Rhythm, a Quantitative In Vivo PET Study
}

\author{
Heli Savolainen, ${ }^{1}$ Peter Meerlo, ${ }^{2}$ Philip H. Elsinga, ${ }^{1}$ Albert D. Windhorst, ${ }^{3}$ Rudi A.J.O. Dierckx, ${ }^{1}$ \\ Nicola A. Colabufo, ${ }^{4,5}$ Aren van Waarde, ${ }^{1}$ and Gert Luurtsema ${ }^{1,6}$
}

Received 27 June 2016; accepted 8 August 2016; published online 6 September 2016

\begin{abstract}
The blood-brain barrier (BBB) contributes to brain homeostasis by protecting the brain from harmful compounds. P-glycoprotein (P-gp) is one of the major efflux transporters at the BBB. In the present study, we assessed whether (1) P-gp function in the brain is constant or fluctuates across the day and (2) if it is affected by sleep deprivation. Four groups of rats were PET scanned with a radiolabeled P-gp substrate $\left[{ }^{18} \mathrm{~F}\right] \mathrm{MC} 225$, each at a different moment of the 12-h light-dark cycle to study diurnal variations: early sleep phase (ZT3), late sleep phase (ZT9), early active phase (ZT15), and late active phase (ZT21). In two additional groups, controls were allowed to sleep normally while experimental animals were sleep-deprived for $10 \mathrm{~h}$ in a slowly rotating drum during the sleep phase. Kinetic modeling with a one-tissue compartment model fit resulted for all brain regions in 1.2-1.8fold higher distribution volumes $\left(V_{T}\right)$ at ZT15 than at other time points. $V_{T^{-v a l u e s}}$ at ZT3, ZT9, and ZT21 were not significantly different from each other. Regional tracer distribution volumes in controls and sleep-deprived animals were also not significantly different. Our results indicate that $\mathrm{P}$-gp function in rats displays a daily rhythm with reduced function at the beginning of the active phase. This rhythm is not dependent on sleep since acute sleep deprivation had no effect. Knowing the diurnal variation of P-gp function could be important for the design of PET studies and for choosing the correct administration time for P-gpdependent drugs.
\end{abstract}

KEYWORDS: circadian rhythm; efflux transporter; $\left[{ }^{18} \mathrm{~F}\right] \mathrm{MC} 225$; pharmacokinetic modeling; sleep deprivation.

\section{INTRODUCTION}

P-glycoprotein (P-gp) is expressed on the luminal side of endothelial cells in the blood-brain barrier (BBB) $(1,2)$. This transporter protects the brain by pumping a wide variety of substances back into the blood. P-gp function is not constant but can be modulated by a wide variety of endogenous $(3,4)$ and exogenous factors (5-7). However, little is known about physiological fluctuations in P-gp function across the day in

\footnotetext{
${ }^{1}$ Department of Nuclear Medicine and Molecular Imaging, University of Groningen, University Medical Center Groningen, Hanzeplein 1, 9713 GZ, Groningen, Netherlands.

${ }^{2}$ Groningen Institute for Evolutionary Life Sciences, University of Groningen, Nijenborgh 7, 9747 AG, Groningen, Netherlands.

${ }^{3}$ Department of Radiology and Nuclear Medicine, VU University Medical Center Amsterdam, De Boelelaan 1085 C, 1081 HV, Amsterdam, Netherlands.

${ }^{4}$ Dipartimento di Farmacia-Scienze del Farmaco, Università degli Studi di Bari, via Orabona 4, 70125, Bari, Italy.

${ }^{5}$ Biofordrug slr, via Orabona 4, 70125, Bari, Italy.

${ }^{6}$ To whom correspondence should be addressed. (e-mail: g.luurtsema@umcg.nl)
}

relation to the circadian rhythm. Such fluctuations could be important for selecting the optimal time of the day for application of therapeutic drugs. By selecting the appropriate hour of administration for a drug which is a (weak) substrate for P-gp, the ratio between beneficial effect and undesired side effects could be optimized. Many CNS drugs have some affinity for P-gp, e.g., certain anticancer drugs, antidepressants, and HIV-protease inhibitors (8-11).

Most processes in the mammalian body display a 24-h rhythm, from molecular processes to behavior. Many of these rhythms are coordinated by an endogenous circadian clock that is located in the suprachiasmatic nuclei $(\mathrm{SCN})$ of the hypothalamus (12). Ultimately, rhythms in the regulation of gene expression, protein synthesis, hormone levels, or neurotransmitter release could affect P-gp expression and function. Moreover, the endogenous circadian clock might not only affect P-gp function through direct physiological mechanisms, but a daily rhythm in P-gp function might also be related to sleep, which is itself under control of the $\operatorname{SCN}(13,14)$.

Recent data have indicated that the clearance of harmful substances from the brain may be directly related to the sleep state. Such substances include the neurodegenerative marker 
amyloid- $\beta$ (Aß) which is a substrate for $\mathrm{P}$-gp (15, 16). Cerebral accumulation of $A \beta$-plaques is a hallmark, and probably also the major cause, of Alzheimer's disease (AD) (17). In humans, both a shorter duration and a reduced quality of sleep result in increased accumulation of $A \beta$ in the brain (18). In mice, natural sleep is associated with a $60 \%$ increase in the volume of the cerebral interstitial space, resulting in an increase in convective exchange of cerebrospinal fluid with interstitial fluid, which causes an increased rate of $A B$ clearance during sleep (19). Another study showed that acute $(6 \mathrm{~h})$ sleep deprivation in wild-type mice and chronic (20 h daily during 21 days) sleep deprivation in an AD mouse model cause a substantial increase in the $A \beta$ plaque burden in the brain (20). Although these studies did not involve specific measurements of the activity of P-gp, the data suggest that the observed fluctuations in $A \beta$ clearance may be related to an altered function of this efflux pump.

In the present study, we used in vivo positron emission tomography (PET) imaging and a substrate radiotracer $\left[{ }^{18} \mathrm{~F}\right] \mathrm{MC} 225(21,22)$ to investigate whether P-gp function in the brain is constant or if it fluctuates across the day. Decreased function of P-gp should correlate with an increased uptake of $\left[{ }^{18} \mathrm{~F}\right] \mathrm{MC} 225$, since after passive diffusion into the brain, the tracer would be less effectively effluxed. We scanned four groups of rats each at a different specific zeitgeber time (ZT, hours after light onset) during the 24-h cycle. Since P-gp function may be regulated by the sleepwake cycle rather than the time of the day, we also investigated whether P-gp function is affected by sleep deprivation. A group of rats was scanned after $10 \mathrm{~h}$ of acute sleep deprivation during the normal sleep phase. The data of these animals were compared to those of a control group which was allowed to sleep normally.

\section{MATERIALS AND METHODS}

\section{Animals and Housing}

Male outbred Sprague-Dawley rats $(388 \pm 35$ g, $12-$ 14 weeks) were obtained from Harlan (Horst, Netherlands). Rats were acclimatized at least 2 weeks in the Central Animal Facility of the University Medical Center Groningen before starting the experiments. Rats were housed in groups of two in a 12-h light-dark regime and had access to standard laboratory food (RMH-B, AB Diets, Woerden, Netherlands) and water ad libitum. Animal experiments were approved by the Institutional Animal Care and Use Committee of the University of Groningen (DEC 6456D) and were in accordance with the Animal Welfare Act of the European Communities Council Directive.

\section{Experimental Groups}

In the first experiment, we determined if there is a daily rhythm in P-gp function by scanning four groups of rats each at a different time during the light-dark cycle. Since rats are nocturnal animals, the light phase is for them the main sleep period and the dark phase the main period of activity. The four groups were scanned at ZT3 (early sleep phase, group 1, $n=$ 8), ZT9 (late sleep phase, group 2, $n=8$ ), ZT15 (early active phase, group $3, n=8$ ), and ZT21 (late active phase, group 4, $n=6)$. Animals in groups 1 and 2 had a light phase from 07:00 to 19:00 and a dark phase from 19:00 to 07:00, whereas for groups 3 and 4, light and dark were reversed. Thus, scan times at ZT3 and ZT9 for groups 1 and 2 and ZT15 and ZT21 for groups 3 and 4 both corresponded to 10:00 and 16.00, respectively.

In the second experiment, we assessed whether a daily rhythm in P-gp function might be due to the alternation of sleep and wakefulness. Two additional groups of rats were scanned at the end of the sleep phase. One group could sleep normally (group 5, $n=7$ ), while the other group (group 6, $n=$ 7) was sleep deprived for most of the light phase (10 h), which in rats is the main sleep phase. Sleep deprivation was achieved by placing an animal in a slowly rotating drum (diameter $40 \mathrm{~cm}$, rotation speed $40 \mathrm{~cm} / \mathrm{min}$ ) at the beginning of the sleep cycle $(23,24)$. A control animal was placed in a non-rotating drum for $10 \mathrm{~h}$. Food and water were available $\mathrm{ad}$ libitum inside the wheels. All animals in groups 5-6 were adapted to non-rotating drums $2 \times 45 \mathrm{~min}$ before the experiments.

\section{PET Radiotracer}

To assess P-gp function, we performed small animal PET with the preclinically validated radiotracer $\left[{ }^{18} \mathrm{~F}\right] \mathrm{MC} 225(5-(1-$ (2- $\left[{ }^{18} \mathrm{~F}\right]$ fluoroethoxy))-[3-(6,7-dimethoxy-3,4-dihydro- $1 H$ isoquinolin-2-yl)-propyl]-5,6,7,8-tetrahydronaphthalen). The tracer was synthesized as previously described (21). In the daily rhythm study, the same batch of $\left[{ }^{18} \mathrm{~F}\right] \mathrm{MC} 225$ was used in the morning and afternoon scans (radiochemical yield $5.2 \pm$ $1.6 \%$ calculated from end of bombardment of $\left[{ }^{18} \mathrm{~F}\right] \mathrm{F}^{-}$). Specific radioactivity at the end of tracer synthesis was $>200 \mathrm{GBq} / \mu \mathrm{mol}$ and radiochemical purity $>98 \%$. Tracer quality was checked before both scans to confirm the stability of $\left[{ }^{18} \mathrm{~F}\right] \mathrm{MC} 225$ ( $>6 \mathrm{~h}$ ). During the PET scans, $1 \mathrm{~mL}$ of radiotracer in saline solution was injected with $18 \pm 5 \mathrm{MBq}$ of radioactivity. In the morning scans, on average $14 \mathrm{ng}$ of non-radioactive MC225 was injected, in the afternoon scans $121 \mathrm{ng}$, and in the sleep deprivation study $107 \mathrm{ng}$.

\section{PET Imaging and Ex Vivo Biodistribution}

Rats were anesthetized with 5\% isoflurane in medical air, and anesthesia was maintained with $1.5-2 \%$ of isoflurane. Body temperature was maintained with a heating pad which was connected to an electronic temperature controller $\left(38^{\circ} \mathrm{C}\right)$. A cannula was inserted into a femoral artery in order to collect blood samples during the scan. A femoral vein was also cannulated for tracer injection. Rats (two at a time) were positioned in the microPET camera (microPET Focus 220, Siemens Medical Solutions, Malvern, USA), and a transmission scan was acquired using a ${ }^{57} \mathrm{Co}$ point source. The radiotracer was injected at a constant speed of $1 \mathrm{~mL} / \mathrm{min}$ using an infusion pump. Data acquisition for a dynamic PET scan of 60-min duration was started simultaneously with the tracer injection. Arterial blood samples (volume $0.1 \mathrm{~mL}$ ) were collected every $10 \mathrm{~s}$ during the first minute and at $1.5,2$, $3,5,7.5,10,15,30$, and $60 \mathrm{~min}$. Collected blood was replaced by heparinized saline. A $25-\mu \mathrm{L}$ aliquot of whole blood was extracted from each sample for radioactivity measurement. The remainder of each sample was centrifuged at $3460 \times g$ for 
$5 \mathrm{~min}$, and $25 \mu \mathrm{L}$ of plasma was taken for radioactivity measurement. The radioactivity in blood and plasma was measured with a gamma counter (LKB Wallac, Turku, Finland). Radioactivity in plasma was expressed as standardized uptake value (SUV), which is defined as [plasma activity concentration $(\mathrm{MBq} / \mathrm{mL})] /[$ injected dose $(\mathrm{MBq}) /$ body weight (g) $]$. Radioactive metabolites of $\left[{ }^{18} \mathrm{~F}\right] \mathrm{MC} 225$ in plasma were measured with thin-layer chromatography (radio-TLC) as described earlier (22). After the scan, rats were perfused with $40 \mathrm{~mL}$ of ice-cold PBS. Some peripheral organs were collected for biodistribution analysis. Organs were weighed, and radioactivity was measured with a gamma counter. Radioactivity in tissue $(\mathrm{MBq} / \mathrm{g})$ was divided by radioactivity in plasma to express the values as tissue-to-plasma ratio.

\section{PET Image Reconstruction and Analysis}

The list-mode data from the emission scan were reconstructed into 21 frames $(6 \times 10,4 \times 30,2 \times 60,1 \times 120,1 \times 180$, $4 \times 300$, and $3 \times 600 \mathrm{~s})$. Emission sinograms were iteratively reconstructed (OSEM 2D, four iterations and 16 subsets) after being normalized and corrected for attenuation and decay of radioactivity. The PET images were analyzed using PMOD v3.5 software (PMOD Technologies, Zürich, Switzerland). Each PET image was automatically co-registered with an MRI template of the rat brain (which is included in the PMOD software package) using rigid matching. Predefined brain regions from the template were selected as volumes of interest (VOI). Blood and metabolite-corrected plasma radioactivity $(\mathrm{Bq} / \mathrm{mL})$ was used as input functions for the kinetic modeling. Tracer distribution volume $\left(V_{T}\right)$, the tissueto-plasma radiotracer concentration ratio at equilibrium, was calculated using a one-tissue compartment model (1TCM) fit for each VOI. Data were weighted for frame duration, and the blood volume in the brain was fixed to $5 \%$ (22).

\section{Statistical Analysis}

Unless otherwise mentioned, data are presented as mean \pm standard error of the mean (SEM). One way analysis of variance (ANOVA) with Bonferroni correction was used to assess the differences between three or more groups and a two-tailed unpaired $t$ test for comparisons between two groups. A $p$ value of less than 0.05 was considered statistically significant. IBM SPSS Statistics version 22 (Armonk, USA) was used for the analysis.
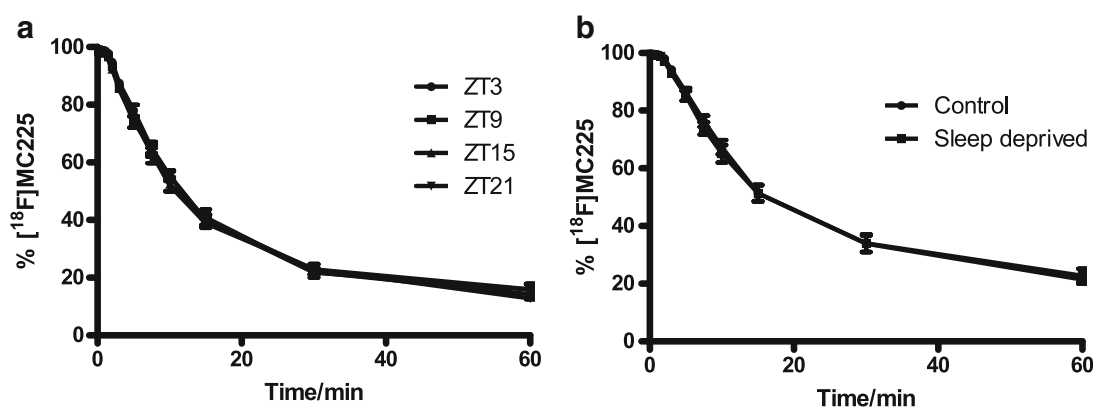

Fig. 1. Fraction of plasma radioactivity representing parent $\left[{ }^{18} \mathrm{~F}\right] \mathrm{MC} 225$ as a function of

time measured in radio-TLC in the a daily rhythm $(n=6-8$ per group) and b sleep deprivation study ( $n=7$ per group)

\section{RESULTS}

\section{Daily Rhythm} pons $(p=0.035)$. observed at ZT15.

\section{Sleep Deprivation}

Radioactive metabolites of the injected PET tracer in plasma were measured using radio-TLC. The rate of tracer metabolism did not differ between groups (Fig. 1a). Metabolite-corrected plasma time-activity curves (SUV) at ZT15 were significantly different from those acquired at ZT3 and ZT9 ( $p=0.026$, and $p<0.001$, respectively, Fig. 2a) when area under the curve (AUC) 0-60 min was compared. Plasma activities were the lowest, and tracer uptake in brain tissue was the highest at ZT15 (Fig. 3). For kinetic modeling, a 1TCM was fitted to the cerebral time-activity data. The 1TCM has been identified as the preferred kinetic model for analysis of $\left[{ }^{18} \mathrm{~F}\right] \mathrm{MC} 225 \mathrm{PET}$ data (22). Tracer distribution volume $\left(V_{T}\right)$ in all analyzed brain regions was 1.2-1.8-fold higher at ZT15 than at other time points (Fig. 3). $V_{T}$-values at ZT3, ZT9, and ZT21 were not significantly different from each other. Examples of $V_{T}$-images are presented in Fig. 4. $V_{T}$ is calculated as $K_{1} / k_{2}$, from a 1TCM fit. The influx parameter $K_{1}$ largely determined the daily pattern of $V_{T}$ as $K_{1}$ displayed the same variation (Table I). $K_{1}$ at ZT15 was on average 1.4fold higher than at other time points. Efflux parameter $k_{2}$ values were similar in all groups. Significant differences in $k_{2}$ values (between ZT3 and ZT21) were observed only in the

Tissue samples of several P-gp expressing peripheral organs were collected at 60 -min post-injection (p.i.) to see if tracer uptake in these organs displays the same diurnal rhythm as was observed in the brain. This was indeed the case for the liver, adrenal gland, kidney, pancreas, and testes (Fig. 5). The highest tracer tissue-to-plasma ratios were again

Significant differences between the two groups in the sleep deprivation study were not observed. Radiotracer metabolism ( $\mathrm{AUC}_{0-60}$ min, Fig. 1b), plasma SUV-curves $\left(\mathrm{AUC}_{0-60} \mathrm{~min}\right.$, Fig. 2b), and regional tracer distribution volumes (Fig. 6a) in controls and sleep-deprived animals were not significantly different. The regional pattern of tracer distribution was the same as was reported previously: highest in cortical areas and lowest in the pons (22). $K_{1}$ and $k_{2}$-values appeared to be higher in sleep-deprived animals than 

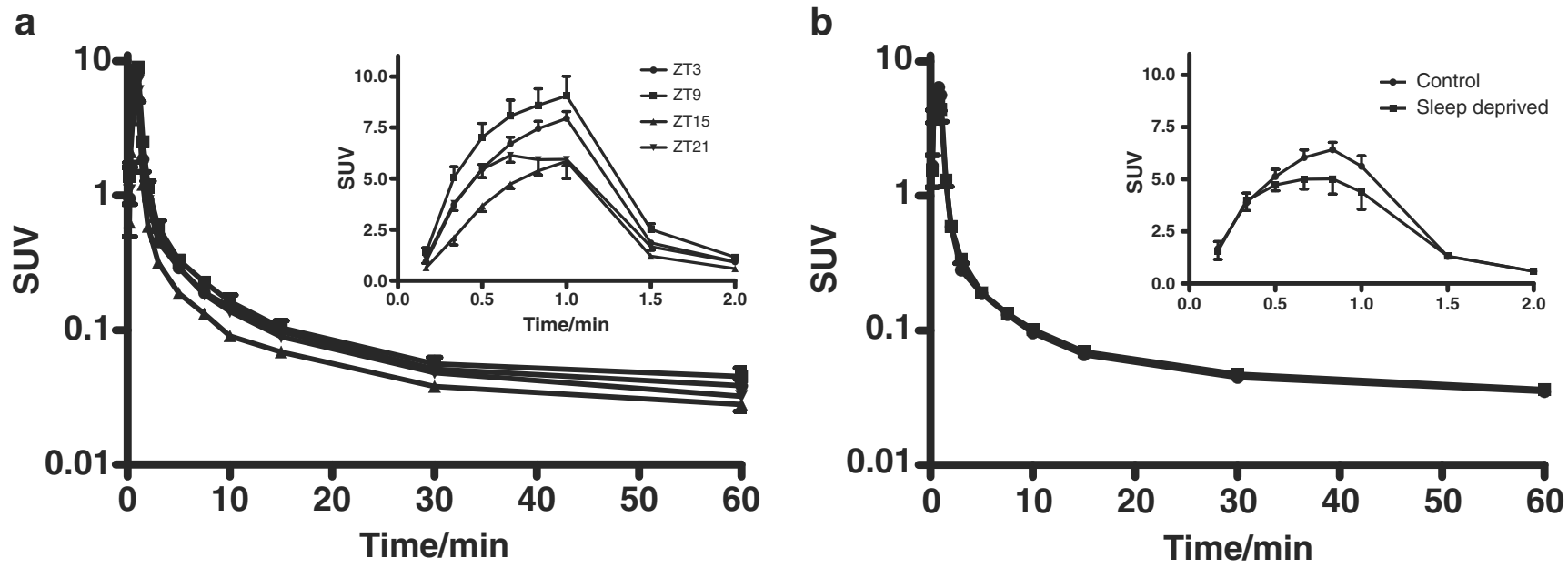

Fig. 2. Metabolite-corrected plasma radioactivity expressed as SUV in the a daily rhythm ( $n=6-8$ per group) and b sleep deprivation study ( $n=7$ per group). The figure inset provides an expanded view of the initial $2 \mathrm{~min}$

controls, but these differences were not statistically significant (Table I). The biodistribution of radioactivity in peripheral organs did not show any significant difference between sleepdeprived animals and controls (Fig. 6b).

\section{DISCUSSION}

In the current study, we investigated diurnal fluctuations of P-gp activity in the brain and the effect of sleep deprivation in rats, using $\left[{ }^{18} \mathrm{~F}\right] \mathrm{MC} 225$ and PET imaging. Brain uptake $\left(V_{T}\right)$ of a P-gp substrate $\left[{ }^{18} \mathrm{~F}\right] \mathrm{MC} 225$ was the highest in the early dark phase (ZT15), which is the active phase in these animals. Brain uptake of the tracer at other time points of the light-dark cycle (ZT3, ZT9, ZT21) was on average 1.5-fold lower. Since P-gp is an efflux transporter, the increased uptake at ZT15 means that P-gp function is reduced at this time of day compared to the other time points. The observed difference is not related to the time-dependent differences of tracer metabolism since metabolite curves in rat plasma at all the time points of the diurnal cycle were identical (Fig. 1a). In many brain regions, the lowest tracer uptake was observed at ZT9 (Fig. 3), indicating that the greatest change in P-gp function occurs at the transition from sleep phase to active phase.

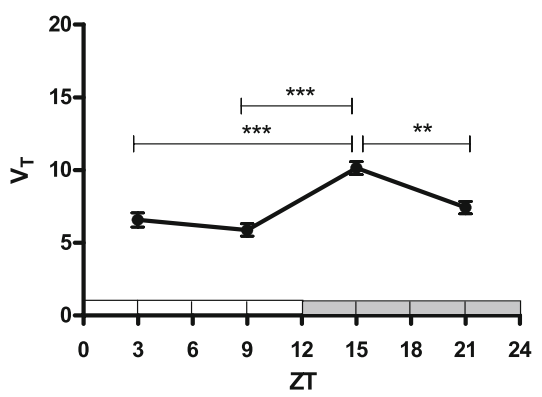

d

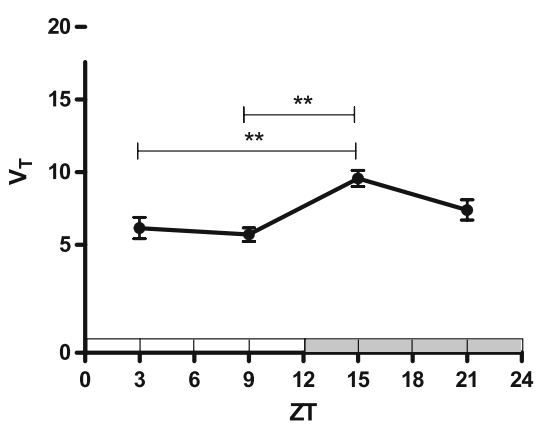

b

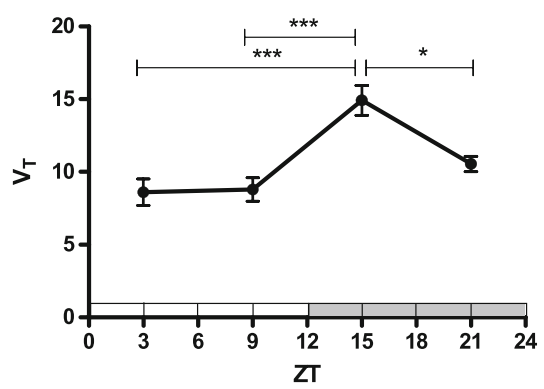

$\mathbf{e}$

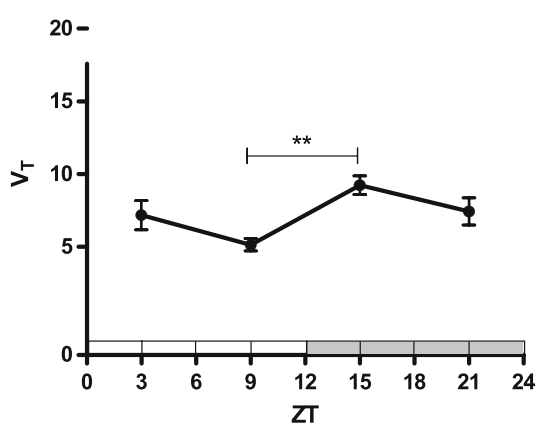

C

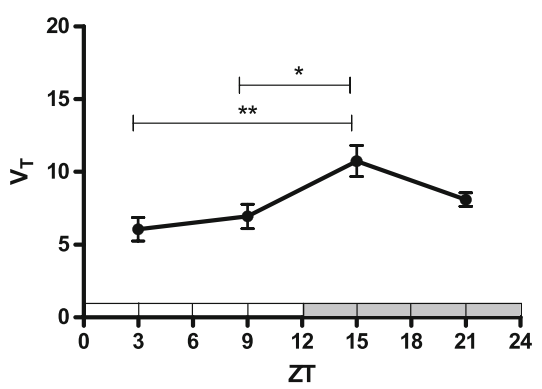

f

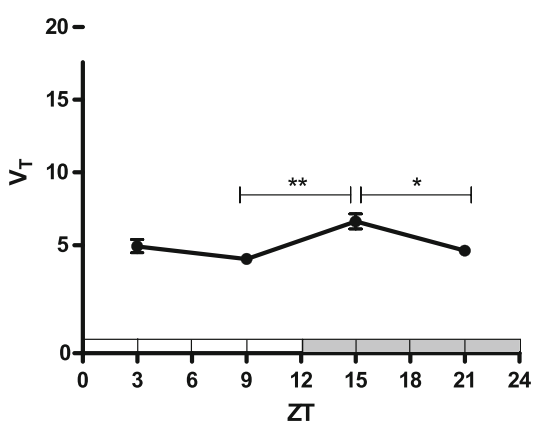

Fig. 3. Regional tracer distribution volumes $\left(V_{T}\right)$ in the daily rhythm study calculated with one-tissue compartment model fit $(n=6-8$ per group). Significant differences are marked with horizontal capped lines $(* p<0.05, * * p<0.01, * * * p<0.001)$. Figure panels concern different brain regions, viz. (a) whole brain, (b) cortex, (c) striatum, (d) hippocampus, (e) cerebellum and (f) pons 


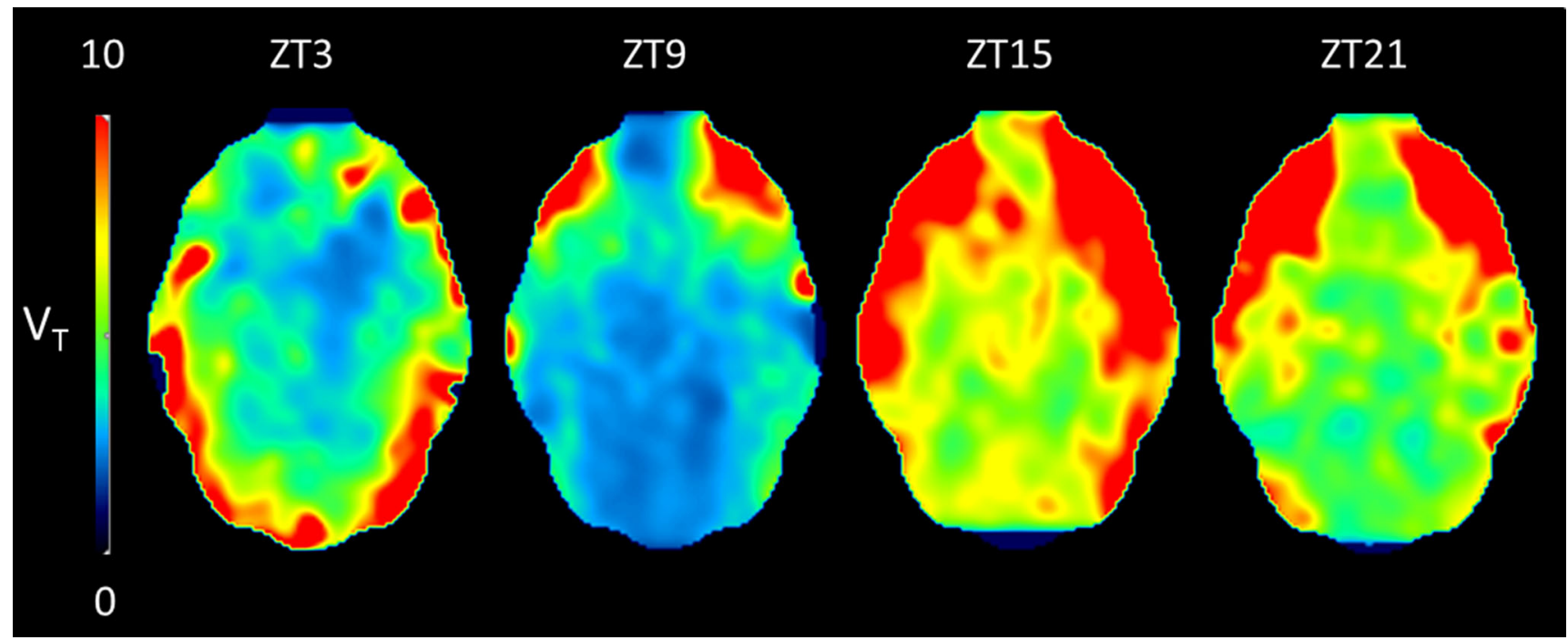

Fig. 4. Examples of parametric $V_{T}$-images in each group of the daily rhythm study (ZT3, ZT9, ZT15, and ZT21). Images were generated using a 1TCM (Alpert) fit in the PXMOD module of the PMOD software

In contrast to the findings of the diurnal rhythm study, we did not observe any difference in regional cerebral tracer uptake between sleep-deprived and control animals after $10 \mathrm{~h}$ of acute sleep deprivation (during the light phase which is the main sleep phase of rats). Therefore, our results suggest that $\mathrm{P}$-gp function in rats displays a daily rhythm with reduced function at the beginning of the active phase which is not dependent on sleep. P-gp function may be modulated by the endogenous circadian clock via other processes than sleep, such as rhythms in neurotransmitters, cytokines, or hormones.

One limitation in our study is the use of anesthesia. Since we cannot perform microPET scans with naturally sleeping or naturally awake rats, all animals were anesthetized with isoflurane during the surgical procedures and scans. All animals had been under anesthesia for about an hour prior to the start of the scan, and they were kept under anesthesia during the scan. Thus, one could argue that changes in P-gp function as a consequence of $10 \mathrm{~h}$ of sleep deprivation might partially disappear during the period of anesthesia which precedes the PET scan. However, after $10 \mathrm{~h}$ of sleep deprivation during the sleep phase, rats need not 1 , but 6 to $8 \mathrm{~h}$ of recovery sleep to fully dissipate the accumulated sleep debt, as reflected by prolonged elevations of EEG slow-wave deltapower (26). From the perspective of sleep debt, it seems unlikely that the brief period of sleep induced by anesthesia could dissipate all effects of sleep deprivation. Thus, our data suggest that acute sleep deprivation has no major effect on $\mathrm{P}$ gp function in the brain.

Our observation of a rhythm in P-gp function in the brain appears to be in accordance with several studies which have shown rhythms of P-gp mRNA or protein expression in other organs. However, the reported shape of these rhythms varies between tissues and studies. For example, a 24-h rhythm in mRNA expression of the Abcbla (1 member of a 3-membered P-gp gene family) (25) was observed in C57BL/6J mouse liver and intestines (27). mRNA expression in the liver peaked at ZT12-ZT16 and at ZT8-ZT12 in the intestines. However, in the kidney, where P-gp is also expressed (25), no daily rhythm in mRNA was found. In addition, mRNA expression of the other members of the Pgp gene family, $A b c b 1 b$ and $A b c b 4$, did not exhibit

Table I. Rate constants obtained with one-tissue compartment model fit using whole blood and metabolite-corrected plasma radioactivity as input (mean $\pm \mathrm{SD}, n=6-8$ per group)

\begin{tabular}{|c|c|c|c|c|c|c|c|}
\hline Parameter & Region & ZT3 & ZT9 & ZT15 & ZT21 & Control & Sleep deprived \\
\hline \multirow[t]{6}{*}{$K_{1}$} & Whole brain & $0.089 \pm 0.018$ & $0.079 \pm 0.018$ & $0.13 \pm 0.018$ & $0.10 \pm 0.011$ & $0.11 \pm 0.026$ & $0.13 \pm 0.035$ \\
\hline & Cortex & $0.084 \pm 0.020$ & $0.071 \pm 0.017$ & $0.13 \pm 0.020$ & $0.099 \pm 0.008$ & $0.11 \pm 0.025$ & $0.12 \pm 0.033$ \\
\hline & Striatum & $0.085 \pm 0.021$ & $0.086 \pm 0.017$ & $0.13 \pm 0.018$ & $0.11 \pm 0.016$ & $0.10 \pm 0.021$ & $0.12 \pm 0.032$ \\
\hline & Hippocampus & $0.087 \pm 0.020$ & $0.077 \pm 0.017$ & $0.13 \pm 0.024$ & $0.11 \pm 0.014$ & $0.10 \pm 0.024$ & $0.12 \pm 0.032$ \\
\hline & Cerebellum & $0.10 \pm 0.022$ & $0.091 \pm 0.024$ & $0.15 \pm 0.023$ & $0.11 \pm 0.011$ & $0.14 \pm 0.032$ & $0.16 \pm 0.043$ \\
\hline & Pons & $0.10 \pm 0.016$ & $0.099 \pm 0.020$ & $0.15 \pm 0.024$ & $0.13 \pm 0.022$ & $0.13 \pm 0.030$ & $0.15 \pm 0.030$ \\
\hline \multirow[t]{6}{*}{$k_{2}$} & Whole brain & $0.014 \pm 0.003$ & $0.014 \pm 0.003$ & $0.013 \pm 0.002$ & $0.014 \pm 0.001$ & $0.018 \pm 0.003$ & $0.021 \pm 0.003$ \\
\hline & Cortex & $0.010 \pm 0.003$ & $0.0085 \pm 0.003$ & $0.0087 \pm 0.002$ & $0.0094 \pm 0.0009$ & $0.014 \pm 0.003$ & $0.016 \pm 0.003$ \\
\hline & Striatum & $0.015 \pm 0.002$ & $0.013 \pm 0.004$ & $0.013 \pm 0.003$ & $0.014 \pm 0.002$ & $0.020 \pm 0.005$ & $0.022 \pm 0.003$ \\
\hline & Hippocampus & $0.015 \pm 0.003$ & $0.014 \pm 0.004$ & $0.014 \pm 0.003$ & $0.015 \pm 0.002$ & $0.019 \pm 0.003$ & $0.022 \pm 0.002$ \\
\hline & Cerebellum & $0.016 \pm 0.006$ & $0.018 \pm 0.005$ & $0.016 \pm 0.003$ & $0.016 \pm 0.004$ & $0.022 \pm 0.002$ & $0.026 \pm 0.004$ \\
\hline & Pons & $0.021 \pm 0.004$ & $0.025 \pm 0.004$ & $0.023 \pm 0.003$ & $0.027 \pm 0.003$ & $0.026 \pm 0.004$ & $0.029 \pm 0.002$ \\
\hline
\end{tabular}


a

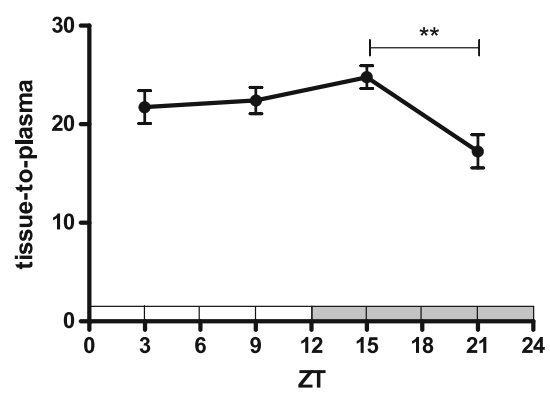

d

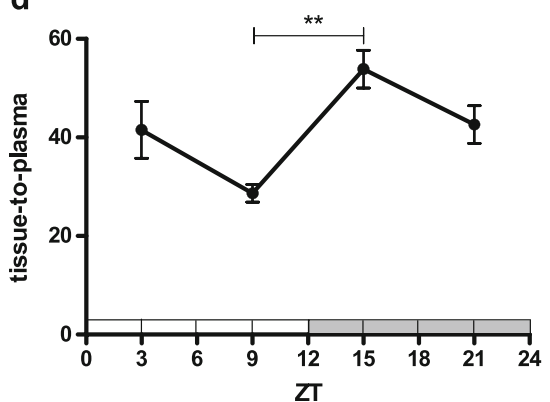

b

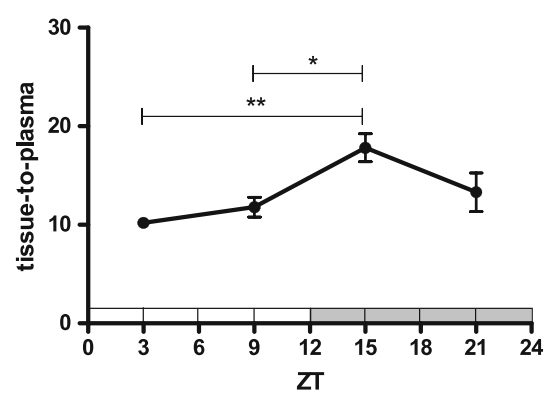

e

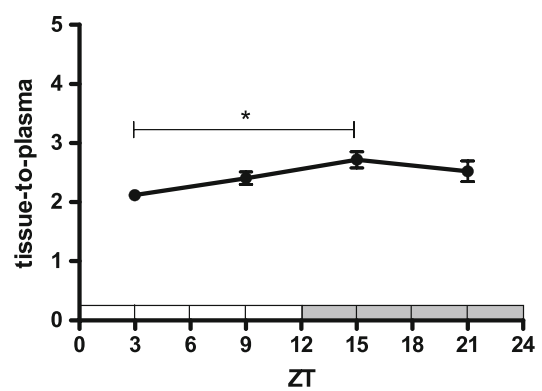

C

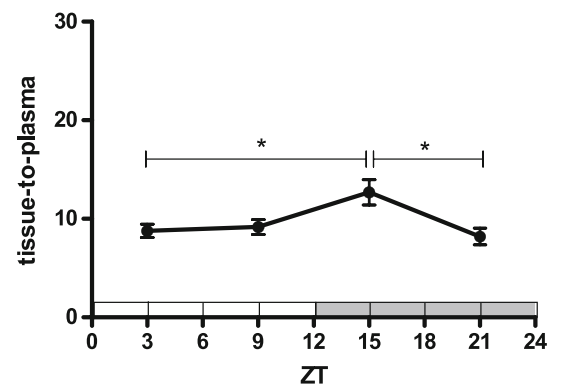

Fig. 5. Ex vivo biodistribution data for the daily rhythm study expressed as tissue-to-plasma ratios of radioactivity at $60 \mathrm{~min}$ p.i. $(n=6-8$ per group). Significant differences are marked with horizontal capped lines $(* p<0.05, * * p<0.01, * * * p<0.001)$. Figure panels concern different peripheral organs, viz. (a) liver, (b) adrenal glands, (c) kidney, (d) pancreas and (e) testes

significant 24-h variation in any of the tissues investigated. Levels of P-gp protein also exhibited a daily rhythm in the intestine, with a peak occurring in the latter half of the sleep phase (ZT8), but not in the liver or kidney. In another study, Abcbla mRNA expression in mouse liver was maximal at ZT17, and 2.9-fold higher than the lowest expression which was observed at ZT0 (28). In mouse ileum, mRNA expression of Abcbla peaked at ZT10, and Pgp protein expression displayed almost the same pattern (29). So, all the studies in mice reported the peak of P-gp mRNA and protein expression in the intestine to be in the late sleep phase and in the liver in the early or mid-active phase. In contrast in rats, intestinal secretion of the P-gp substrates talinolol and losartan was greater during nocturnal activity (ZT13-ZT15) than daytime rest (ZT1-ZT3)
(30). P-gp rhythm has been investigated also in daytimeactive cynomolgus monkeys (31). mRNA levels of $A b c b 1$ oscillated in both the liver and intestine, but the daily variation was higher in the intestine being highest at ZT2 (early active phase) and lowest at ZT8 (mid-active phase). In contrast, protein expression in the liver did not show any obvious circadian oscillation. In the small intestine, an opposite behavior was noted to mRNA and protein expression, the latter showing a decrease at the early active phase and an increase from the mid-active phase to the early sleep phase. Thus, gene expression, protein expression, and protein function may not show a direct correlation.

Since different organs were reported to show a different pattern of diurnal changes, also P-gp function at the BBB may show its own distinct rhythm. The only
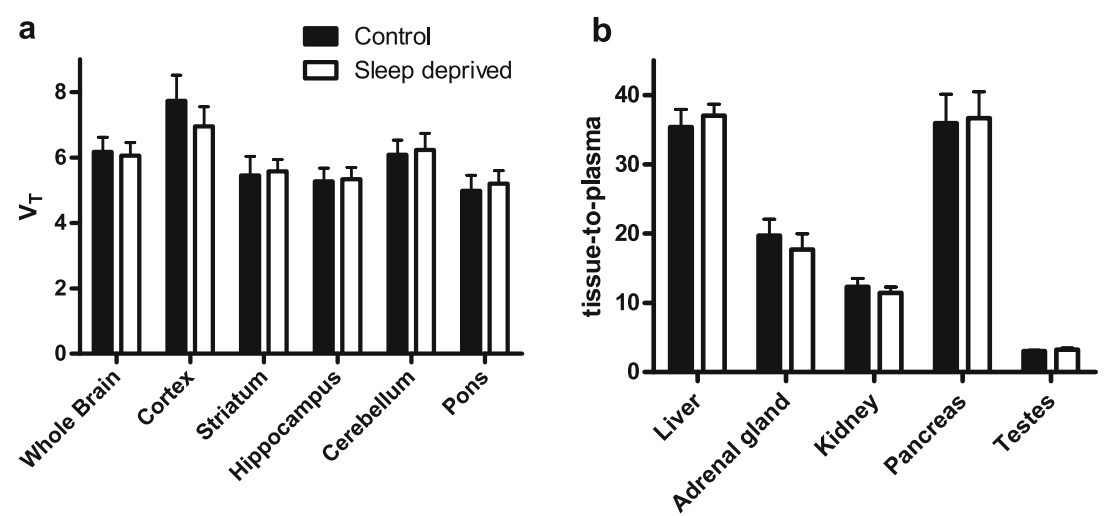

Fig. 6. a Regional tracer distribution volumes and $\mathbf{b}$ ex vivo biodistribution data for the sleep deprivation study ( $n=7$ per group) expressed as tissue-to-plasma ratios of radioactivity at $60 \mathrm{~min}$ p.i. Significant differences between control and sleep-deprived animals were not observed 
publication which addressed diurnal rhythmicity of P-gp function in the brain, found on average 2-fold higher P-gp mediated quinidine transport in rat brain throughout the active period than during daytime rest (32). Our PET data is conflicting to these findings, as we found that the P-gp function in the brain is the weakest during the early active period. $\left[{ }^{18} \mathrm{~F}\right] \mathrm{MC} 225$ and quinidine may have different transporter selectivity, which could in part explain this result. $\left[{ }^{18} \mathrm{~F}\right] \mathrm{MC} 225$ has been evaluated for its specificity in transporter knockout mice and in rats with inhibitors (21, 22). It is selective to P-gp and has only negligible affinity to breast cancer resistance protein (Bcrp), which is another efflux transporter at the $\mathrm{BBB}$ and belongs to the same family as P-gp. Quinidine has been found to be specific to Pgp in knockout mice (33). The earlier study, however, did find that the cerebrospinal fluid (CSF) flux was almost twice as high during the resting period (ZT8) compared to active period (ZT20) (32). We also used different techniques for measuring the P-gp substrates, Kervezee et al. ex vivo measurements and microdialysis, whereas we used PET imaging. The strength of PET is that it measures regional distribution of a tracer non-invasively and quantitatively in time-dependent manner, but it measures only total radioactivity and cannot distinguish between bound and unbound tracer concentrations.

The data reported in this paper may have important implications for the design of PET studies of P-gp function in human subjects. The light phase is for humans the active phase, as opposed to rodents. Thus, it may matter whether humans are scanned in the morning or in the afternoon, since differences in P-gp function between the early and late active phase can be expected. Treatment groups should not be scanned at different time points during the day (e.g., group 1 in the morning and group 2 in the afternoon) in order to avoid differences which are related to diurnal variation rather than to treatment. If scanning all subjects at the same time point is not feasible, the scans should be randomized across the day. In a test-retest study in humans performed with a P-gp substrate $\left[{ }^{11} \mathrm{C}\right]$ verapamil, subjects were scanned initially at $10: 30$ and a second time at 14:00. In the scans performed in the afternoon, a significantly lower tracer uptake $\left(V_{T}\right)$ in gray matter of the entire brain was observed by Logan graphical analysis (34). The authors explained this observed difference by changes of cerebral blood volume, but our data suggest that diurnal variation of $\mathrm{P}-\mathrm{gp}$ activity could also play a role. In rats, on average, $20 \%$ higher cerebral blood flow has been measured during the active cycle compared to the sleep cycle $(35,36)$. We scanned rats at two separate time points during the active phase, but only at ZT15, the tracer uptake was significantly increased, although the cerebral blood flow remains high throughout the active cycle. Therefore, it is unlikely that variations in blood flow alone can explain the increase of $\left[{ }^{18} \mathrm{~F}\right] \mathrm{MC} 225$ uptake in the early active phase.

Daily fluctuations of P-gp activity in human beings could have important implications for therapy design. The P-gp rhythmicity could be exploited depending on the question whether the aim is to limit or increase the CNS distribution of a drug. A potential strategy to improve therapeutic effectiveness and to minimize side effects would be careful timing of a CNS drug administration, so that the drug is administered at the time point when P-gp function is minimal. If our results in rats would be translatable to humans, the most optimal time point to take a P-gpdependent CNS medication would be early in the morning, at the onset of our daily activities. Treatment scheduling according to a circadian or other rhythm, known as "chronotherapy," is already considered important for the treatment of various disorders such as epilepsy, (37) cardiac diseases $(38,39)$, and cancer (40-42). These schedules are based on the observation that treatment-related parameters such as the occurrence of seizures, heart rate, blood pressure, the activity of vasodilating hormones, and cellular proliferation show diurnal rhythms.

\section{CONCLUSION}

P-gp function in the rat brain displays a daily rhythm with a reduced function at the beginning of the active phase. This rhythm is not dependent on sleep since acute sleep deprivation had no effect. P-gp function may be modulated by the endogenous circadian clock via other processes than sleep, such as rhythms in neurotransmitters, cytokines, or hormones.

\section{ACKNOWLEDGMENTS}

This study was funded by the Dutch Technology Foundation STW (project number 11741). We thank Jürgen Sijbesma for his help during the microPET scans, Ronald Boellaard for suggestions in the data analysis, and David Vállez García for help in making parametric images.

Open Access This article is distributed under the terms of the Creative Commons Attribution 4.0 International License (http://creativecommons.org/licenses/by/4.0/), which permits unrestricted use, distribution, and reproduction in any medium, provided you give appropriate credit to the original author(s) and the source, provide a link to the Creative Commons license, and indicate if changes were made.

\section{REFERENCES}

1. Schinkel AH. P-Glycoprotein, a gatekeeper in the blood-brain barrier. Adv Drug Deliv Rev. 1999;36(2, 3):179-94.

2. Liu $X$, Chen $C$, Smith BJ. Progress in brain penetration evaluation in drug discovery and development. J Pharmacol Exp Ther. 2008;325(2):349-56.

3. Sukhai M, Piquette-Miller M. Regulation of the multidrug resistance genes by stress signals. J Pharm Pharm Sci. 2000;3(2):268-80.

4. Miller DS, Bauer B, Hartz AMS. Modulation of P-glycoprotein at the blood-brain barrier: opportunities to improve central nervous system pharmacotherapy. Pharmacol Rev. 2008;60:196-209.

5. Chan GNY, Saldivia V, Yang Y, Pang H, de Lannoy I, Bendayan $\mathrm{R}$. In vivo induction of P-glycoprotein expression at the mouse blood-brain barrier: an intracerebral microdialysis study. J Neurochem. 2013;127(3):342-52.

6. Moerman L, Wyffels L, Slaets D, Raedt R, Boon P, De Vos F. Antiepileptic drugs modulate P-glycoproteins in the brain: a mice study with ${ }^{11}$ C-desmethylloperamide. Epilepsy Res. 2011;94(1, 2):18-25. 
7. Yousif S, Chaves C, Potin S, Margaill I, Scherrmann J-M, Declèves X. Induction of P-glycoprotein and Bcrp at the rat blood-brain barrier following a subchronic morphine treatment is mediated through NMDA/COX-2 activation. J Neurochem. 2012;123(4):491-503.

8. Benish RL, Rodriguez B, Zimmerman PA, Mehlotra RK. Comparative description of haplotype structure and genetic diversity of MDR1 (ABCB1) in HIV-positive and HIVnegative populations. Infect Genet Evol. 2010;10(1):60-7.

9. Breedveld P, Beijnen JH, Schellens JHM. Use of P-glycoprotein and BCRP inhibitors to improve oral bioavailability and CNS penetration of anticancer drugs. Trends Pharmacol Sci. 2006;27(1):17-24.

10. Doran A, Obach RS, Smith BJ, Hosea NA, Becker S, Callegari $\mathrm{E}$, et al. The impact of P-glycoprotein on the disposition of drugs targeted for indications of the central nervous system: evaluation using the mdr1a/1b knockout mouse model. Drug Metab Dispos. 2005;33(1):165-74.

11. O'Brien FE, Dinan TG, Griffin BT, Cryan JF. Interactions between antidepressants and P-glycoprotein at the blood-brain barrier: clinical significance of in vitro and in vivo findings. $\mathrm{Br} \mathrm{J}$ Pharmacol. 2012;165(2):289-312.

12. Hastings MH, Reddy AB, Maywood ES. A clockwork web: circadian timing in brain and periphery, in health and disease. Nat Rev Neurosci. 2003;4(8):649-61.

13. Mistlberger RE. Circadian regulation of sleep in mammals: role of the suprachiasmatic nucleus. Brain Res Brain Res Rev. 2005;49:429-54.

14. Beersma DGM, Gordijn MCM. Circadian control of the sleepwake cycle. Physiol Behav. 2007;90(2, 3):190-5.

15. Ohtsuki S, Ito $\mathrm{S}$, Terasaki $\mathrm{T}$. Is P-glycoprotein involved in amyloid- $\beta$ elimination across the blood-brain barrier in Alzheimer's disease? Clin Pharmacol Ther. 2010;88(4):443-5.

16. Cirrito JR, Deane R, Fagan AM, Spinner ML, Parsadanian M, Finn MB, et al. P-glycoprotein deficiency at the blood-brain barrier increases amyloid- $\beta$ deposition in an Alzheimer disease mouse model. J Clin Invest. 2005;115(11):3285-90.

17. Castello MA, Soriano S. On the origin of Alzheimer's disease. Trials and tribulations of the amyloid hypothesis. Ageing Res Rev. 2014;13(1):10-2.

18. Bilgel M, Zhou Y, Wong DF, Ferrucci L, Resnick SM. Selfreported sleep and $\beta$-amyloid deposition in community-dwelling older adults. JAMA Neurol. 2013;70(12):1537-43.

19. Xie L, Kang H, Xu Q, Chen MJ, Liao Y, Thiyagarajan M, et al. Sleep drives metabolite clearance from the adult brain. Science. 2013;342(6156):373-7.

20. Kang J, Lim MM, Bateman RJ, Lee JJ, Smyth LP, Cirrito JR, et al. Amyloid- $\beta$ dynamics are regulated by orexin and the sleepwake cycle. Science. 2009;326(5955):1005-7.

21. Savolainen H, Cantore M, Colabufo NA, Elsinga PH, Windhorst $\mathrm{AD}$, Luurtsema G. Synthesis and preclinical evaluation of three novel fluorine-18 labeled radiopharmaceuticals for Pglycoprotein PET imaging at the blood-brain barrier. Mol Pharm. 2015;12(7):2265-75.

22. Savolainen H, Windhorst AD, Elsinga PH, Cantore M, Colabufo NA, Willemsen ATM, et al. Evaluation of $\left[{ }^{18} \mathrm{~F}\right] \mathrm{MC} 225$ as a PET radiotracer for measuring P-glycoprotein function at the bloodbrain barrier in rats; kinetics, metabolism, and selectivity. J Cereb Blood Flow Metab. 2016. doi:10.1177/0271678X16654493

23. Meerlo P, Koehl M, van der Borght K, Turek FW. Sleep restriction alters the hypothalamic-pituitary-adrenal response to stress. J Neuroendochrinology. 2002;14(5):397-402.

24. Novati A, Roman V, Cetin T, Hagewoud R, den Boer JA, Luiten PG, et al. Chronically restricted sleep leads to depression-like changes in neurotransmitter receptor sensitivity and neuroendocrine stress reactivity in rats. Sleep. 2008;31(11):1579-85.

25. Schinkel AH, Smit JJM, van Tellingen O, Beijnen JH, Wagenaar E, van Deemter L, et al. Disruption of the mouse mdr1a Pglycoprotein gene leads to a deficiency in the blood-brain barrier and to increased sensitivity to drugs. Cell. 1994;77(4):491-502.

26. Tobler I, Borbely AA. Sleep EEG in the rats as a function of prior waking. Electroencephalogr Clin Neurophysiol. 1986;64(1):74-6.

27. Ando H, Yanagihara H, Sugimoto K, Hayashi Y, Tsuruoka S, Takamura T, et al. Daily rhythms of P-Glycoprotein expression in mice. Chronobiol Int. 2005;22(4):655-65.

28. Zhang YK, Yeager RL, Klaassen CD. Circadian expression profiles of drug processing genes and transcription factors in mouse liver. Drug Metab Dispos. 2008;37(1):106-15.

29. Murakami Y, Higashi Y, Matsunaga N, Koyanagi S, Ohdo S. Circadian clock-controlled intestinal expression of the multidrug-resistance gene mdr1a in mice. Gastroenterology. 2008;135(5):1636-44.

30. Okyar A, Dressler C, Hanafy A, Baktir G, Lemmer B, SpahnLangguth $\mathrm{H}$. Circadian variations in exsorptive transport: in situ intestinal perfusion data and in vivo relevance. Chronobiol Int. 2012;29(4):443-53.

31. Iwasaki M, Koyanagi S, Suzuki N, Katamune C, Matsunaga N, Watanabe $\mathrm{N}$, et al. Circadian modulation in the intestinal absorption of P-glycoprotein substrates in monkeys. Mol Pharmacol. 2015;88(1):29-37.

32. Kervezee L, Hartman R, van den Berg D-J, Shimizu S, EmotoYamamoto $\mathrm{Y}$, Meijer $\mathrm{JH}$, et al. Diurnal variation in $\mathrm{P}$ glycoprotein-mediated transport and cerebrospinal fluid turnover in the brain. AAPS J. 2014;16(5):1029-37.

33. Kodaira H, Kusuhara H, Ushiki J, Fuse E, Sugiyama Y. Kinetic analysis of the cooperation of P-glycoprotein (P-gp/Abcb1) and breast cancer resistance protein (Bcrp/Abcg2) in limiting the brain and testis penetration of erlotinib, flavopiridol, and mitoxantrone. J Pharmacol Exp Ther. 2010;333(3):788-96.

34. Lubberink M, Luurtsema G, van Berckel BNM, Boellaard R, Toornvliet R, Windhorst AD, et al. Evaluation of tracer kinetic models for quantification of P-glycoprotein function using $(R)$ $\left[{ }^{11} \mathrm{C}\right]$ verapamil and PET. J Cereb Blood Flow Metab. 2007;27(2):424-33.

35. Wauschkuhn CA, Witte K, Gorbey S, Lemmer B, Schilling L. Circadian periodicity of cerebral blood flow revealed by laserDoppler flowmetry in awake rats: relation to blood pressure and activity. Am J Physiol Hear Circ Physiol. 2005;289(4):H1662-8.

36. Endo Y, Jinnai K, Endo M, Fujita K, Kimura F. Diurnal variation of cerebral blood flow in rat hippocampus. Stroke. 1990;21(10):1464-9.

37. Glauser TA, Pippenger CE. Chronotherapy in the treatment of epilepsy. ChronoPhysiology Ther. 2014;4:109-23.

38. Takeda N, Maemura K. Cardiovascular disease, chronopharmacotherapy, and the molecular clock. Adv Drug Deliv Rev. 2010;62(9, 10):95666.

39. Singh V, Deshpande A. The emergence of time programmed drug delivery system: chronotherapy of cardio vascular diseases. Int J Pharm Pharm Sci. 2014;6(10):56-60.

40. Kondratov R. Circadian clock and cancer therapy: an unexpected journey. Ann Med. 2014;46(4):189-90.

41. Fu L, Lee CC. The circadian clock: pacemaker and tumor suppressor. Nat Rev. 2003;3(5):350-61.

42. Ortiz-Tudela E, Mteyrek A, Ballesta A, Innominato PF, Levi F. Cancer chronotherapeutics: experimental, theoretical, and clinical aspects. Circadian Clocks. 2013;217:261-88. 